\section{(- OPEN ACCESS}

\title{
Prognosis and NT-proBNP in heart failure patients with preserved versus reduced ejection fraction
}

\author{
Khibar Salah, ${ }^{\oplus 1,2}$ Susan Stienen, ${ }^{\oplus 1,3}$ Yigal M Pinto, ${ }^{1}$ Luc W Eurlings, ${ }^{4}$ Marco Metra, ${ }^{5}$ \\ Antoni Bayes-Genis, ${ }^{6}$ Valerio Verdiani, ${ }^{7}$ Jan G P Tijssen, ${ }^{1}$ Wouter E Kok ${ }^{1}$
}

- Additional material is published online only. To view please visit the journal online (http://dx.doi.org/10.1136/ heartjnl-2018-314173).

${ }^{1}$ Heart Centre, Department of Clinical and Experimental Cardiology, Amsterdam Cardiovascular Sciences, Amsterdam UMC, University of Amsterdam, Amasterdam, The Netherlands

${ }^{2}$ Department of Radiology and Nuclear Medicine, Radboud

University Medical Centre Nijmegen, Nijmegen, The Netherlands

${ }^{3}$ INSERM, Centre d'Investigation Cliniques Plurithématique, Université de Lorraine, CHRU de Nancy, Nancy, France

${ }^{4}$ Department of Cardiology, VieCuri Medical Centre, Venlo,

The Netherlands

${ }^{5}$ Department of Medical and

Surgical Specialties, Radiological

Sciences and Public Health,

Cardiology, University of

Brescia, Brescia, Italy

${ }^{6}$ Department of Cardiology,

CIBERCV, Hospital Universitari

Germans Trias i Pujol, Barcalona,

Spain

7 Department of Internal

Medicine and Emergency, Careggi University Hospital,

Florence, Italy

Correspondence to Khibar Salah, AMC, Amsterdam 1105 AZ, The Netherlands; k.salah@amc.uva.n

Received 21 September 2018 Revised 18 January 2019 Accepted 24 January 2019

Published Online First

8 April 2019

\section{Check for updates}

(C) Author(s) (or their employer(s)) 2019. Re-use permitted under CC BY. Published by BMJ.

To cite: Salah K, Stienen S, Pinto YM, et al. Heart 2019;105:1182-1189.

\section{ABSTRACT}

Background We assessed the prognostic significance of absolute and percentage change in N-terminal proB-type natriuretic peptide (NT-proBNP) levels in patients hospitalised for acute decompensated heart failure with preservedejection fraction (HFpEF) versus heart failure with reduced ejection fraction (HFrEF).

Methods Patients with left ventricular ejection fraction $\geq 50 \%$ were categorised as HFpEF $(n=283)$, while those with $<40 \%$ as were categorised as HFrEF $(n=776)$. Prognostic values of absolute and percentage change in NT-proBNP levels for 6 months all-cause mortality after discharge were assessed separately in patients with HFpEF and HFrEF by multivariable adjusted Cox regression analysis. Comorbidities were compared between heart failure groups.

Results Discharge NT-proBNP levels predicted outcome similarly in HFpEF and HFrEF: for any 2.7-factor increase in NT-proBNP levels, the HR for mortality was 2.14 for HFpEF (95\% Cl 1.48 to 3.09) and 1.96 for HFrEF (95\% Cl 1.60 to 2.40). Mortality prediction was equally possible for NT-proBNP reduction of $\leq 30 \%$ (HR $4.60,95 \% \mathrm{Cl}$ 1.47 to 14.40 and HR $3.36,95 \% \mathrm{Cl} 1.93$ to 5.85 for HFpEF and HFrEF, respectively) and for $>30 \%-60 \%$ (HR 3.28, 95\% Cl 1.07 to 10.12 and $\mathrm{HR} 1.79,95 \% \mathrm{Cl}$ 0.99 to 3.26 , respectively), compared with mortality in the reference groups of $>60 \%$ reductions in NT-proBNP levels. Prognostically relevant comorbidities were more often present in patients with HFpEF than patients with HFrEF in low $(\leq 3000 \mathrm{pg} / \mathrm{mL})$ but not in high $(>3000 \mathrm{pg} /$ $\mathrm{mL}$ ) NT-proBNP discharge categories.

Conclusions Our study highlights-after demonstrating that NT-proBNP levels confer the same relative risk information in $\mathrm{HFpEF}$ as in $\mathrm{HFrEF}$ - the possibility that comorbidities contribute relatively more to prognosis in patients with HFpEF with lower NTproBNP levels than in patients with HFrEF.

\section{INTRODUCTION}

While in-hospital mortality is lower in heart failure with preserved ejection fraction (HFpEF) than in heart failure patients with reduced left ventricular ejection fraction (HFrEF), ${ }^{1-3}$ mortality after discharge has been reported to be similar to that of patients with HFrEF in studies on patients after hospitalisations for acute decompensated heart failure (ADHF). ${ }^{3-7}$ Recent studies show that in a more stabilised phase, mortality is lower in patients with HFpEF than in patients with HFrEF in populations consisting either of a mix of inpatient and outpatient heart failure patients ${ }^{8}$ or in populations with exclusively outpatients with stable heart failure. ${ }^{9}$ In the latter situation, there are also lower mortality rates compared with the mortality rate after hospitalisations for heart failure.

The prognostic value of absolute levels of B-type natriuretic peptide (BNP) and N-terminal pro-Btype natriuretic peptide (NT-proBNP) has been well established for patients hospitalised for ADHF with either type of heart failure but also specifically for patients with HFpEF. ${ }^{6}{ }^{10-18}$ Prognostic information of a single measurement of natriuretic peptide levels has been specifically investigated in the comparison between patients with HFpEF and HFrEF and has been reported to be equal for the two heart failure types either at admission or at discharge. ${ }^{6710}$ Also, the recently published multicentre study in combined inpatient and outpatient setting with a follow-up of 2 years showed that NT-proBNP levels at clinical stabilisation are strongly and similarly related to survival in heart failure regardless of ejection fraction and that a given level of NT-proBNP portends the same risk of death in HFpEF and HFrEF. ${ }^{8}$

The suggestion that a single baseline or discharge measurement of BNP or NT-proBNP may be equally useful in risk-stratifying patients with $\mathrm{ADHF}$ irrespective of the type of heart failure confronts us with the difficulty of explaining why prognosis is similar between the two groups, first of all because natriuretic peptide plasma levels are almost half in HFpEF compared with HFrEF. ${ }^{6} 719$ Second, absolute levels of NT-proBNP at admission or at discharge are interpreted as single values, but the assumption may then be that the reduction in NT-proBNP during hospitalisation would be equal in both types of heart failure. A third issue is that the risk assessment of hospitalised heart failure patients with the use of natriuretic peptides is done with relative risks, leaving unexplained that lower discharge natriuretic peptides in patients with HFpEF are associated with similar outcomes as in patients with $\mathrm{HFrEF}$ who have higher discharge levels. Finally, even if it can be shown that single values of NT-proBNP are predictive of outcome without distinction between HFpEF and HFrEF, the attainability of these levels may become the factor that determines whether HFpEF or HFrEF have a similar prognosis on a population level.

Therefore, we assessed the prognostic contribution of absolute levels of NT-proBNP and percentage change in NT-proBNP levels in patients with HFpEF and HFrEF hospitalised for ADHF. In 
addition, we assessed the attainability of several (absolute and relative) discharge NT-proBNP targets in patients with HFpEF and HFrEF. Finally, we assessed the frequencies of prognostically relevant comorbidities in patients with HFpEF and HFrEF for low and high discharge NT-proBNP categories.

\section{METHODS}

\section{Source/study populations}

The presently studied population consisted of five of seven cohorts from the European collaboration on acute decompensated heart failure database with exact data available on left ventricular ejection fraction. ${ }^{11}$ Details on the search strategy, source gathering and explicit information on data collection for these prospective ADHF cohorts have been reported previously. ${ }^{11}$ In addition to these five cohorts, data from the Can NT-proBNP guided therapy during hospital admission for acute decompensated heart failure reduce mortality and readmissions? (PRIMA II) trial was used for the analyses. ${ }^{18} 20$ The PRIMA II was a randomised controlled trial investigating the effect of NT-proBNP-guided (targeting a $>30 \%$ NT-proBNP at discharge) versus conventional therapy in patients with ADHF, demonstrating a neutral effect. ${ }^{182021}$

The study population for the present study was assembled by the following criteria: (1) patients were hospitalised because of clinically validated $\mathrm{ADHF}^{22}$ (2) they were discharged alive, (3) left ventricular ejection fraction (LVEF) measurements were performed during admission and (4) NT-proBNP levels were available at admission and/or at discharge. For the present study, patients with heart failure were categorised into three groups: those with LVEF $\geq 50 \%$ were categorised as HFpEF, those with $<40 \%$ were categorised as HFrEF and patients with LVEF range of $40 \%-49 \%$ were defined as heart failure with midrange EF (HFmrEF), based on the definition of heart failure in the recently published European Society of Cardiology (ESC) guidelines. ${ }^{22}$ The PRIMA II study was funded by the Dutch Heart Foundation grant 2010B97, and NT-proBNP kits were supplied by Roche Diagnostics. ${ }^{18} 2021$ The authors are solely responsible for the design and conduct of this study, all study analyses, the drafting and editing of the paper and its final contents.

\section{Statistical analysis}

Our primary endpoint for this study was 6-month all-cause mortality; the secondary endpoint was a composite endpoint of 6-month cardiovascular readmission/all cause mortality. To illustrate the relation between both endpoints (6-month all-cause mortality and 6-month cardiovascular readmission/allcause mortality) and heart failure types, we plotted the survival curve that is adjusted for clinical variables (age $\geq 75$ years, peripheral oedema at admission, systolic blood pressure (SBP) $<115 \mathrm{~mm}$ $\mathrm{Hg}$, hyponatraemia (sodium levels $<135 \mathrm{mmol} / \mathrm{L}$ ) and serum urea levels $\geq 15 \mathrm{mmol} / \mathrm{L})^{1118}$ and performed the log-rank test. For statistical analysis, we used multivariable Cox regression analysis for both primary and secondary endpoints. Categorisation of absolute discharge NT-proBNP levels was done by making quartiles of NT-proBNP levels at discharge among those patients with HFpEF who died, because this creates an equal distribution of the events among the quartiles. This has the statistical advantage of creating quartiles with equal statistical power to identify predictor variables. For practical purpose, we rounded off the quartile boundaries to convenient cut-offs levels. It should be noted that for the calculation of the risk estimate and for the construction of the Kaplan Meier (KM)curves, we did use the whole population. The tertiles for NT-proBNP percentage reduction were calculated using the total cohort and were used instead of a previous dichotomous approach, because of the possible interaction with baseline NT-proBNP levels ${ }^{23}$ so as to discern more than 'only' a 30\% reduction in NT-proBNP levels as prognostically relevant.

The focus of our study was to assess a possible difference in the prognostic contribution of NT-proBNP levels in patients with HFpEF versus patients with HFrEF. ${ }^{22}$ A separate analysis in HFmrEF was not deemed useful, as the main differences were expected between both ends of the spectrum of ejection fractions. For comparison with previous studies, ${ }^{67}$ we performed additional analyses using the 2-log and the natural logarithm scale for NT-proBNP levels at discharge for both endpoints (6-month all-cause mortality and 6-month cardiovascular readmission/all cause mortality). First, univariable Cox proportional hazard regression analysis was performed for both HFpEF and HFrEF using log-transformed NT-proBNP levels at admission and at discharge and a separate analysis using NT-proBNP quartiles at discharge. The univariable model compares the three NT-proBNP categories to the category of NT-proBNP $\leq 1000$, therefore this is the refence category. Thereafter, we excluded the category $1001-3000 \mathrm{pg} / \mathrm{mL}$ from the multivariable model in a backward selection fashion, because it did not contain any prognostic information in our univariable model. A multivariable Cox-regression model was then made for both endpoints (6-month all-cause mortality and 6-month cardiovascular readmission/all cause mortality) with adjustment for aforementioned factors, which previously demonstrated to be prognostically significant. ${ }^{11}$ Similarly, HRs for relative NT-proBNP changes in categories were calculated in each of the two populations, with adjustment from the same variables. The proportionality of hazard function after transforming NT-proBNP levels was tested by generating time dependent covariates by creating interactions of the predictors and a function of survival time and including them in the model and further by testing for a non-zero slope in a generalised linear regression of the scaled Schoenfeld residuals on functions of time. ${ }^{24}$ Both tests supported the proportional hazards assumption.

As a measure of attainability for a number of preset NT-proBNP levels at discharge and NT-proBNP reduction percentages, the percentage of patients attaining these levels was determined for patients with $\mathrm{HFpEF}$ and HFrEF. Finally, the presence of comorbidities of established prognostic relevance was compared between patients with $\mathrm{HFpEF}$ and patients with $\mathrm{HFrEF}$ in a low $(<3000 \mathrm{pg} / \mathrm{mL})$ and high $(>3000 \mathrm{pg} / \mathrm{mL})$ category of discharge NT-proBNP levels. The Fisher's exact test was used to make a comparison. Normally distributed were compared using the Student's t-test. Other continuous data were compared using the Mann-Whitney U test.

To accommodate for the different cohorts, separate baseline hazard functions were used to adjust for between-study differences. For the multivariable model, we performed multiple imputation pooling algorithms $(n=10)$ to correct for missing values. The imputation method was fully conditional specification with a linear regression for the model for scale variables. Eventually producing output for each 'complete' dataset, plus pooled output. We used these pooled results, which are pooled by Rubin's rules, by taking the average over the parameter estimates from all imputed datasets. All patient, medical history and treatment variables (including outcome variables) were used when creating the multiple imputation data sets.

All probability values were two sided and considered significant if $<0.05$. Statistical analyses were conducted using SPSS V.24.0.0.0. 
Table 1 Baseline characteristics study population

\begin{tabular}{|c|c|c|c|c|c|}
\hline & $\begin{array}{l}\text { Total cohort } \\
(n=1228)\end{array}$ & $\begin{array}{l}\text { HFpEF }(\geq 50 \%) \\
(n=283)\end{array}$ & $\begin{array}{l}\text { HFmrEF }(40 \%-49 \%) \\
(n=169)\end{array}$ & $\begin{array}{l}\operatorname{HFrEF}(<40 \%) \\
(n=776)\end{array}$ & $P$ value \\
\hline Age, years, median (IQR) & $74(64-81)$ & $78(70-83)$ & $76(70-83)$ & $72(61-79)$ & $<0.001$ \\
\hline Age $\geq 75$ years, $\mathrm{n}(\%)$ & $596(49)$ & $182(64)$ & $96(57)$ & $318(41)$ & $<0.001$ \\
\hline Male, n (\%) & $733(60)$ & $104(37)$ & $96(57)$ & $533(69)$ & $<0.001$ \\
\hline History of DM, n (\%) & $376(31)$ & $90(32)$ & $61(36)$ & $225(29)$ & 0.183 \\
\hline History of hypertension, $\mathrm{n}(\%)$ & $659(54)$ & $198(70)$ & $99(59)$ & $362(47)$ & $<0.001$ \\
\hline History of COPD, n (\%) & $206(19)$ & $59(22)$ & $32(20)$ & $115(17)$ & 0.166 \\
\hline Ischaemic aetiology, n (\%) & $548(50)$ & $142(59)$ & $77(50)$ & $329(47)$ & 0.006 \\
\hline Peripheral oedema at admission, $\mathrm{n}(\%)$ & $707(62)$ & $176(67)$ & $89(61)$ & $442(61)$ & 0.155 \\
\hline Rales at admission, $\mathrm{n}(\%)$ & $847(75)$ & $196(75)$ & $115(78)$ & $536(74)$ & 0.492 \\
\hline $\mathrm{SBP}$ at admission, $\mathrm{mm} \mathrm{Hg}$, mean $\pm \mathrm{SD}$ & $135 \pm 32$ & $145 \pm 33$ & $141 \pm 32$ & $130 \pm 30$ & $<0.001$ \\
\hline $\mathrm{DBP}$ at admission, $\mathrm{mm} \mathrm{Hg}$, mean $\mathrm{SD}$ & $81 \pm 22$ & $81 \pm 24$ & $79 \pm 21$ & $81 \pm 20$ & 0.408 \\
\hline Heart rate at admission, bpm, mean \pm SD & $94 \pm 26$ & $91 \pm 27$ & $92 \pm 27$ & $95 \pm 26$ & 0.101 \\
\hline Atrial fibrillation at admission, $\mathrm{n}(\%)$ & $495(43)$ & $140(52)$ & $72(46)$ & $283(39)$ & 0.001 \\
\hline Left ventricular ejection fraction, mean $\pm S D$ & $36 \pm 15$ & $58 \pm 8.8$ & $43 \pm 2.9$ & $26 \pm 7.0$ & $<0.001$ \\
\hline \multicolumn{6}{|l|}{ Laboratories findings, mean \pm SD } \\
\hline Haemoglobin at admission, $\mathrm{mmol} / \mathrm{L}$ & $7.9 \pm 1.2$ & $7.5 \pm 1.3$ & $8.0 \pm 1.2$ & $8.0 \pm 1.2$ & $<0.001$ \\
\hline Serum urea at admission, $\mathrm{mmol} / \mathrm{L}$ & $12 \pm 7.6$ & $12 \pm 7.0$ & $12 \pm 7.7$ & $12 \pm 7.8$ & 0.440 \\
\hline Serum sodium at admission, $\mathrm{mmol} / \mathrm{L}$ & $138 \pm 4.7$ & $138 \pm 4.7$ & $139 \pm 5.1$ & $138 \pm 4.6$ & 0.743 \\
\hline eGFR at admission, $\mathrm{mL} / \mathrm{min} / 1.73 \mathrm{~m}$ & $57 \pm 33$ & $54 \pm 24$ & $55 \pm 28$ & $56 \pm 35$ & 0.611 \\
\hline NT-proBNP at admission, pg/mL, median (IQR) & $6310(3324-11839)$ & $4436(2590-8669)$ & $5254(3037-10868)$ & $7173(4039-13264)$ & $<0.001$ \\
\hline NT-proBNP at discharge, pg/mL, median (IQR) & $3053(1425-6661)$ & $2147(1114-4161)$ & $2743(1351-6047)$ & $3695(1611-7738)$ & $<0.001$ \\
\hline NT-proBNP reduction, \%, median (IQR) & $47(20-70)$ & $47(25-68)$ & $45(17-70)$ & $47(19-70)$ & 0.693 \\
\hline Duration admission, days, median (IQR) & $9(6-14)$ & $7(6-13)$ & $8(5-13)$ & $9(6-15)$ & 0.010 \\
\hline \multicolumn{6}{|l|}{ Discharge medication, $\mathrm{n}(\%)$} \\
\hline Diuretics & $1076(94)$ & $220(92)$ & $144(88)$ & $712(95)$ & 0.417 \\
\hline ACE-inhibitor/ARBs & $748(65)$ & $131(55)$ & $110(67)$ & $507(68)$ & $<0.001$ \\
\hline Beta-blocker & $744(65)$ & $144(61)$ & $105(64)$ & $495(66)$ & 0.252 \\
\hline
\end{tabular}

ARBs, angiotensin II receptor blockers; COPD, chronic obstructive pulmonary disease; DM, diabetes mellitus; DBP, diastolic blood pressure; eGFR, estimated glomerular filtration rate; $\mathrm{HFmrEF}$, heart failure with midrange ejection fraction; $\mathrm{HFpEF}$, heart failure with preserved ejection fraction; $\mathrm{HFrEF}$, heart failure with reduced ejection fraction; NT-proBNP, $\mathrm{N}$-terminal pro-B-type natriuretic peptide; SBP, systolic blood pressure.

\section{RESULTS}

\section{Demographic characteristics}

Patients with HFpEF were significantly older compared with those with HFmrEF and HFrEF with significantly more patients aged $\geq 75$ years (table 1 ). HFpEF was more frequently associated with female sex, hypertension, ischaemic aetiology, higher admission SBP and atrial fibrillation (AF) at admission. Compared with $\mathrm{HFrEF}$ and HFmrEF, HFpEF patients had significantly lower admission haemoglobin levels and lower admission and discharge NT-proBNP levels. The median NT-proBNP reduction percentage during hospitalisation was equal for $\mathrm{HFpEF}$, HFmrEF and HFrEF. At discharge, significantly less HFpEF patients received ACE-inhibitors or angiotensin II receptor blockers (ARBs) compared with patients with HFmrEF and HFrEF, but diuretics and beta-blockers were prescribed with similar frequency. There was no difference in 6-month all-cause mortality (figure 1A) between patients with $\mathrm{HFpEF}, \mathrm{HFmrEF}$ or HFrEF $(14 \%(n=38)$ vs $14 \%(n=23)$ vs $16 \% \quad(n=121)$, respectively, log-rank $=0.60)$. The same pattern was seen for the composite endpoint of 6-month cardiovascular readmission/all-cause mortality ( $42 \%$ vs $40 \%$ vs $45 \%$, respectively, log-rank $=0.45)$. The median NT-proBNP percentage reduction stratified for the four NT-proBNP categories at admission for patients with HFpEF and HFrEF showed the higher the admission NT-proBNP, the higher the median percentage reduction (online supplementary materials). Supplementary figures

\section{NT-proBNP and outcome}

The lower the admission or discharge NT-proBNP levels, the lower the 6-month all-cause mortality rate (figure 2). For the three NT-proBNP percentage reduction categories, 6-month all-cause mortality decreased with higher percentage NT-proBNP reductions for all three heart failure groups.

\section{Cox regression models}

The natural logarithmic scale for NT-proBNP levels at discharge showed that for every 2.7 increase in NT-proBNP level at discharge, the multivariably adjusted HR for 6-month mortality was 2.14 (95\% CI 1.48 to 3.09) in patients with HFpEF and 1.96 (95\% CI 1.60 to 2.40 ) in patients with HFrEF. For the composite endpoint of 6-month cardiovascular readmission/ all-cause mortality, the HR was 1.27 (95\% CI 1.06 to 1.52 ) in patients with HFpEF and 1.39 (95\% CI 1.25 to 1.56) in patients with HFrEF. A $\log 2$ transformation scale for NT-proBNP levels at discharge showed an adjusted HR for 6-month mortality of 1.71 (95\% CI 1.33 to 2.20) for HFpEF, and 1.60 (95\% CI 1.38 to 1.99 ) for HFrEF for every twofold increase in NT-proBNP levels at discharge and an HR of 1.18 (95\% CI 1.04 to 1.34) for HFpEF and 1.26 (95\% CI 1.17 to 1.36 ) for the composite endpoint of 6-month cardiovascular readmission/all-cause mortality.

After adjustment for relevant clinical variables (table 2A), similar significant HRs were found for HFpEF as well as HFrEF 


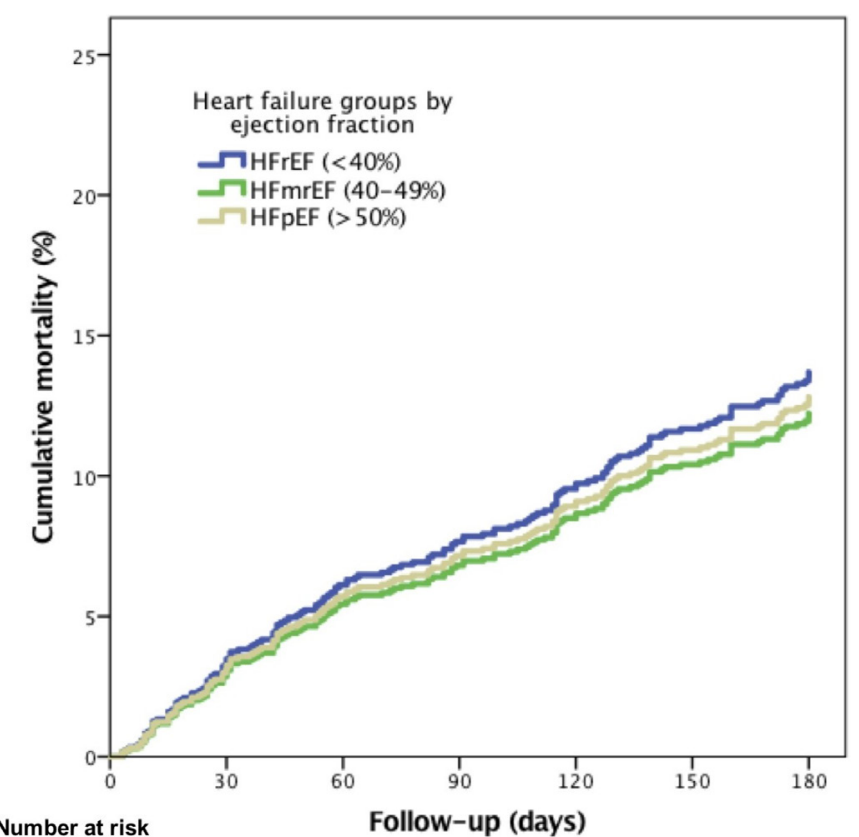

Number at risk Follow-up (days)

$\begin{array}{lrrrrrrr}\text { HFrEF: } & 776 & 744 & 710 & 692 & 663 & 640 & 618 \\ \text { HFmrEF: } & 169 & 163 & 159 & 154 & 151 & 147 & 143 \\ \text { HfpEF: } & 283 & 267 & 257 & 253 & 246 & 243 & 237\end{array}$

Figure 1 Relationship between 6-month all-cause mortality and the three types of heart failure adjusted for age $\geq 75$ years, peripheral edema at admission, systolic blood pressure (SBP) $<115 \mathrm{~mm} \mathrm{Hg}$, hyponatraemia (sodium levels $<135 \mathrm{mmol} / \mathrm{L}$ ) and serum urea levels $\geq 15$ $\mathrm{mmol} / \mathrm{L}$. HFmrEF, heart failure with mid range ejection fraction; HFpEF, heart failure with preserved ejection fraction; HFrEF, heart failure with reduced ejection fraction.

for the highest quartile of absolute NT-proBNP values at discharge (HR 5.68, 95\% CI 2.24 to 14.36 and HR 4.79, $95 \%$ CI 2.76 to 8.33 , respectively). Table $2 \mathrm{~B}$ shows that for HFpEF and HFrEF, both reduction percentage levels of 30\%-60\% (HR $3.28,95 \%$ CI 1.07 to 10.12 and HR 1.79 , CI 0.99 to 3.26 , respectively) as well as $\leq 30 \%$ (HR $4.60,95 \%$ CI 1.47 to 14.40 and HR 3.36, 95\% CI 1.93 to 5.85), respectively) are predictors of mortality compared with a reference of $>60 \%$. The same pattern was seen for both the discharge NT-proBNP levels as well as percentage reduction levels and the composite endpoint of 6-month cardiovascular readmission/all-cause mortality, although with lower HRs.

\section{Attainability of NT-proBNP levels}

For the lowest absolute NT-proBNP target of $<1000 \mathrm{pg} / \mathrm{mL}$, $23 \%$ of patients with HFpEF attained the target (table 3 ) versus $14 \%$ of patients with HFrEF $(p=0.002)$. Also, for the absolute level of $<3000 \mathrm{pg} / \mathrm{mL}$, patients with HFpEF more often attained this target compared with patients with HFrEF (65\% vs $43 \%$, respectively, $\mathrm{p}<0.001)$. No differences for attaining relative reduction levels were found between HFpEF and HFrEF. No significant differences were found between HFpEF and HFrEF in attainability of absolute or relative levels when stratified for admission NT-proBNP categories (online supplementary materials).

\section{NT-proBNP and comorbidities}

Table 4 shows that comorbidities and prognostic factors are evenly distributed among the higher NT-proBNP discharge category in patients with HFpEF and HFrEF (61\% and 60\% of patients, respectively) but are unevenly distributed within the lower discharge NT-proBNP category in patients with HFpEF compared with in patients with HFrEF (37\% of patients versus $24 \%$ of patients, respectively, $\mathrm{p}=0.011$ ). There were no differences in survival between patients with HFpEF and HFrEF as compared in a low $(\leq 3000 \mathrm{pg} / \mathrm{mL})$ or in a high $(>3000 \mathrm{pg} / \mathrm{mL})$ NT-proBNP discharge category in KM analysis (online supplementary materials).

\section{DISCUSSION}

It was previously reported that a doubling of natriuretic peptides at discharge carries a HR of 1.4 for (18 months) mortality, similarly for patients with $\mathrm{HFpEF}$ as for patients with HFrEF. ${ }^{7}$ In our study, for every twofold increase in discharge NT-proBNP, the multivariably adjusted HR for 6-month mortality was 1.71 in patients with HFpEF and 1.60 in patients with HFrEF, which
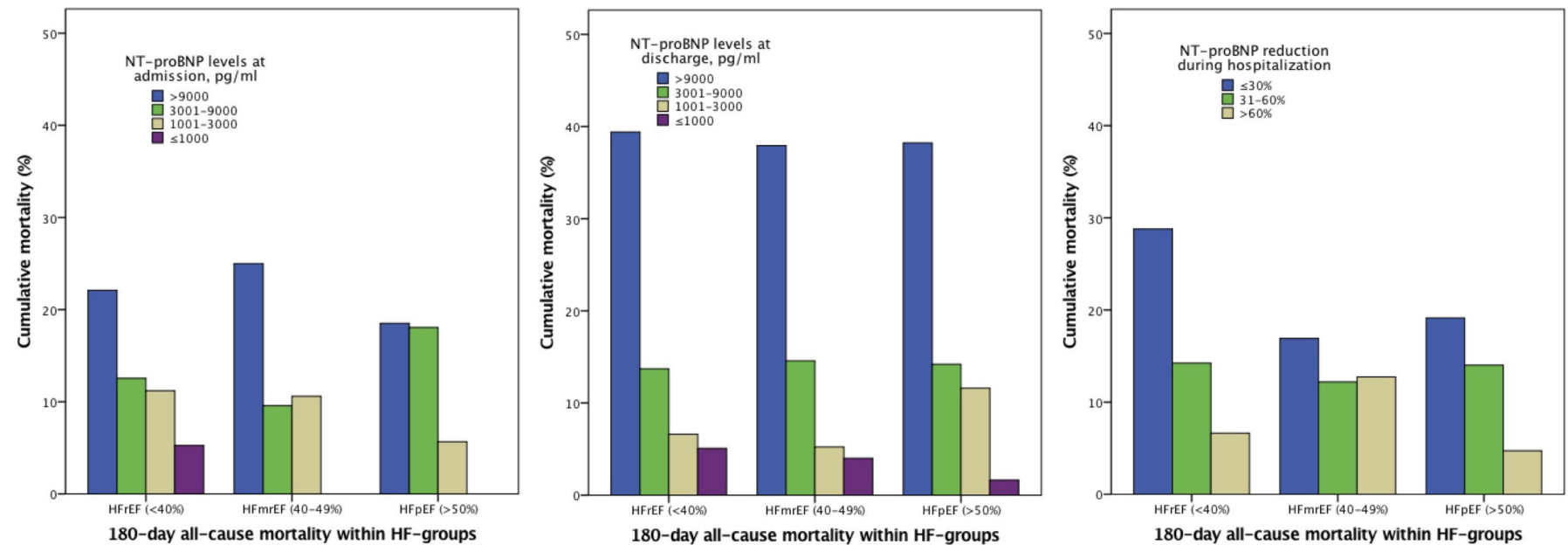

Figure 2 Relationship between 6-month all-cause mortality and the four categories of admission and discharge NT-proBNP levels as well as for the categories of percentage reduction during hospitalisation according to the types of HF. HF, heart failure; HFmrEF, heart failure with midrange ejection fraction; HFpEF, heart failure with preserved ejection fraction; HFrEF, heart failure with reduced ejection fraction NT-proBNP, N-terminal pro-B-type natriuretic peptide. 
Table 2A Cox regression for absolute NT-proBNP levels at discharge and 6-month all-cause mortality

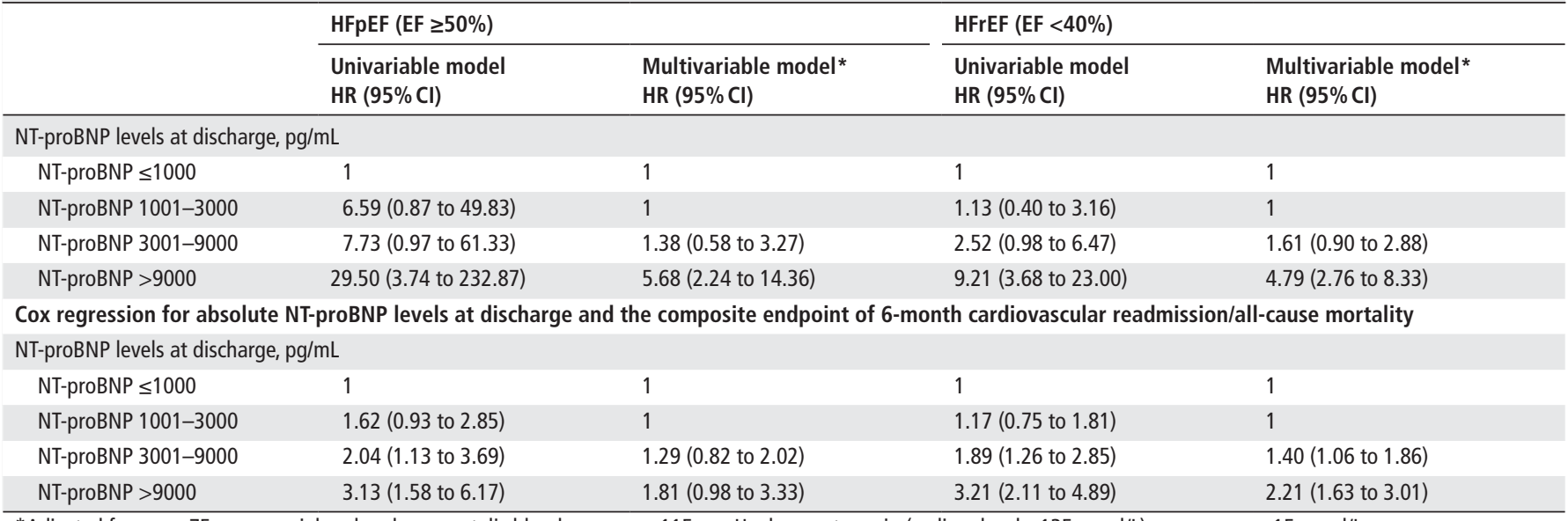

*Adjusted for age $\geq 75$ years, peripheral oedema, systolic blood pressure $\leq 115 \mathrm{~mm} \mathrm{Hg}$, hyponatraemia (sodium level $<135 \mathrm{mmol} / \mathrm{L}$ ), serum urea $\geq 15 \mathrm{mmol} / \mathrm{L}$.

$\mathrm{EF}$, ejection fraction; $\mathrm{HFpEF}$, heart failure with preserved ejection fraction; HFrEF, heart failure with reduced ejection fraction; NT-proBNP, N-terminal pro-B-type natriuretic peptide.

also did not differ significantly from each other between HFpEF and HFrEF. Also, for categories, both for HFpEF as well as HFrEF, only the two highest quartiles of absolute NT-proBNP levels at discharge were predictive for mortality after adjustment for covariables, with similar HRs for patients with HFrEF as for patients with HFpEF. Our study also shows similar adjusted prognostic relative risks in patients with HFpEF and HFrEF for the relative changes in NT-proBNP levels during hospitalisation. In this respect, we confirm previous studies that have reported on equal prognostic significance of either admission ${ }^{6}$ levels or discharge levels of natriuretic peptides in patients with $\mathrm{HFpEF}$ and HFrEF hospitalised for ADHF. ${ }^{70}$

For the prognostic ability of relative changes in NT-proBNP during admissions for heart failure, we also confirm results of a small study demonstrating that changes of $<30 \%$ in NT-proBNP levels during admissions are as predictive of outcome in patients with HFpEF as in a mixed population of patients with $\mathrm{HFpEF}$ and HFrEF. ${ }^{11} 1218$ It is also in line with another report in a mixed population of patients with HFpEF and HFrEF with ADHF that described that a $<50 \%$ reduction in NT-proBNP at discharge was a predictor for 6-month mortality without any significant effect from adjustment by ejection fraction. ${ }^{25}$ In our analysis of three categories of percentage change in NT-proBNP levels, the adjusted multivariable analysis demonstrated a significant and similar contribution to prognosis in patients with $\mathrm{HFpEF}$ and HFrEF for a 30\%-60\% reduction in NT-proBNP as well as for $\mathrm{a} \leq 30 \%$ reduction in NT-proBNP. The results endorse our risk stratification model of a mixed population of patients with ADHF, in which both absolute levels at discharge and NT-proBNP reduction percentage are independent predictors of postdischarge mortality ${ }^{16}$ and also that both measures may be useful as discharge thresholds in patients hospitalised for ADHF. $^{23}$

\section{How to interpret similar outcomes in HFpEF and HFrEF despite lower admission and discharge NT-proBNP levels in HFpEF}

In our study, 6-month mortality was similar between patients with HFpEF and HFrEF, in accordance with other studies on patients with HFpEF and HFrEF after ADHF hospitalisation. ${ }^{3-7}$ However, like numerous other reports, we describe that natriuretic peptide levels are much lower in patients with HFpEF than in patients with HFrEF. ${ }^{6710131526}$ The primary determinant of release of NT-proBNP is myocardial diastolic wall stress which, following the Law of LaPlace, is directly related to the transmural pressure gradient and chamber diameter and inversely related to wall thickness. Therefore, patients with HFpEF are more likely to have lower NT-proBNP levels despite a possible equally high elevated wedge pressure. ${ }^{8} 27$ Since the NT-proBNP

Table 2B Cox regression for NT-proBNP percentage reduction during hospitalisation and 6-month all-cause mortality

\begin{tabular}{|c|c|c|c|c|}
\hline & \multicolumn{2}{|l|}{ HFpEF (EF $\geq 50 \%)$} & \multicolumn{2}{|l|}{ HFrEF $($ EF $<40 \%)$} \\
\hline & $\begin{array}{l}\text { Univariable model } \\
\text { HR }(95 \% \mathrm{Cl})\end{array}$ & $\begin{array}{l}\text { Multivariable model* } \\
\text { HR }(95 \% \mathrm{Cl})\end{array}$ & $\begin{array}{l}\text { Univariable model } \\
\mathrm{HR}(95 \% \mathrm{Cl})\end{array}$ & $\begin{array}{l}\text { Multivariable model* } \\
\text { HR }(95 \% \mathrm{Cl})\end{array}$ \\
\hline \multicolumn{5}{|c|}{ NT-proBNP reduction during hospitalisation } \\
\hline$>60 \%$ & 1 & 1 & 1 & 1 \\
\hline $30 \%-60 \%$ & 3.35 (1.11 to 10.11$)$ & $3.28(1.07$ to 10.12$)$ & 2.23 (1.23 to 4.04$)$ & 1.79 (0.99 to 3.26$)$ \\
\hline$\leq 30 \%$ & 5.01 (1.62 to 15.55$)$ & 4.60 (1.47 to 14.40$)$ & 5.12 (3.00 to 8.73$)$ & $3.36(1.93$ to 5.85$)$ \\
\hline \multicolumn{5}{|c|}{$\begin{array}{l}\text { Cox regression for NT-proBNP percentage reduction during hospitalisation and the composite endpoint of 6-month cardiovascular readmission/all-cause } \\
\text { mortality }\end{array}$} \\
\hline \multicolumn{5}{|c|}{ NT-proBNP reduction during hospitalisation } \\
\hline$>60 \%$ & 1 & 1 & 1 & 1 \\
\hline $30 \%-60 \%$ & 1.51 (0.91 to 2.49$)$ & 1.41 (0.83 to 2.38$)$ & 1.99 (1.48 to 2.69$)$ & 1.78 (1.32 to 2.42$)$ \\
\hline$\leq 30 \%$ & 1.63 (1.03 to 2.60$)$ & 1.69 (1.06 to 2.71$)$ & 2.77 (2.08 to 3.68$)$ & 2.21 (1.63 to 3.00$)$ \\
\hline
\end{tabular}

${ }^{*}$ Adjusted for age $\geq 75$ years, peripheral oedema, systolic blood pressure $\leq 115 \mathrm{~mm} \mathrm{Hg}$, hyponatraemia (sodium level $<135 \mathrm{mmol} / \mathrm{L}$ ), serum urea $\geq 15 \mathrm{mmol} / \mathrm{L}$.

$\mathrm{EF}$, ejection fraction; HFpEF, heart failure with preserved ejection fraction; HFrEF, heart failure with reduced ejection fraction; NT-proBNP, N-terminal pro-B-type natriuretic peptide. 
Table 3 Attainability of absolute and relative NT-proBNP targets for types of heart failure

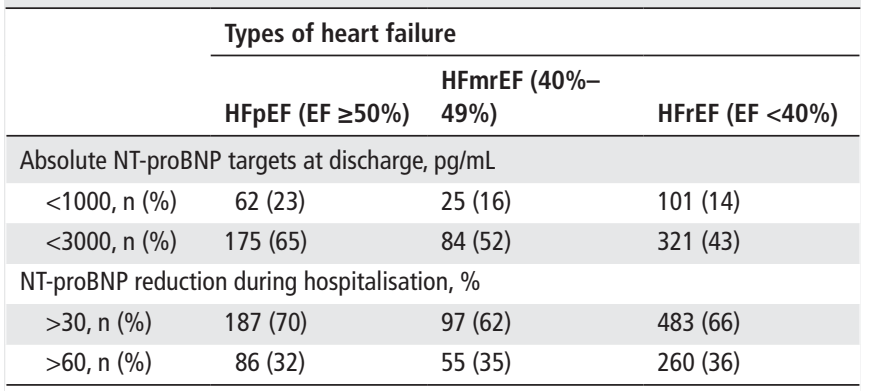

$\mathrm{EF}$, ejection fraction; $\mathrm{HFmrEF}$, heart failure with midrange ejection fraction; $\mathrm{HFpEF}$, heart failure with preserved ejection fraction; HFrEF, heart failure with reduced ejection fraction; NT-proBNP, N-terminal pro-B-type natriuretic peptide.

levels are almost half in patients with HFpEF, the HR of 1.6-1.7 that we found for doubling NT-proBNP levels would lead to an expected reduced mortality in HFpEF with almost a factor 0.60 , that is, from our reported $16 \%$ mortality in HFrEF patients to an expected $10 \%$ in patients with HFpEF. This was obviously not the case in our study, with $16 \%$ vs $14 \%$ mortality in patients with HFrEF versus patients with HFpEF after 6 months. Still, in outpatient populations and in mixed populations of outpatient and patients discharged from hospital, HFpEF patients have lower mortality rates than patients with HFrEF, which would be more in accordance with the lower NT-proBNP levels in patients with HFpEF (MAGGIC and Lam et $a l^{89}$ ).

One of the possible answers to this paradox is that although natriuretic peptides levels are equal indicators of relative risk in patients with HFpEF and HFrEF, it is only a matter of chance finding that the outcomes of patients with HFpEF and HFrEF are alike, because other predictors of death (and possibly mode of death) and also other drivers of high NT-proBNP may be present in uneven distribution in patients with HFpEF and HFrEF ${ }^{16}$ who also differ in many other baseline characteristics.

It was previously suggested that to understand prognosis in patients with HFpEF, absolute NT-proBNP levels in the lower, most frequent, categories are less contributing to prognosis in HFpEF than other prognostic variables such as kidney function or age. ${ }^{28}$ Also in our study, compared with patients with HFrEF, patients with HFpEF have significantly more comorbidities with significantly older patients, more frequently with hypertension, more $\mathrm{AF}$ at admission and lower mean levels of haemoglobin at admission, all of which were previously reported to be of prognostic significance in HFpEF but also in patients with HFrEF. ${ }^{29}$ Our report extends these findings, in that the presence of comorbidities probably precedes rising NT-proBNP levels more in patients with HFpEF than in patients with HFrEF, so that HFpEF patients already seem to have more comorbidities at lower NT-proBNP levels than patients with HFrEF. With higher NT-proBNP levels, the comorbidities and prognostic factors increase but are then as frequently present in HFpEF as in patients with HFrEF. To further investigate the problem of risk prediction with natriuretic peptides in the face of comorbidities, outcome differentiation and/or increasing the number of contributing comorbidities in the prognostic models both seem worthwhile.

\section{Attainability of NT-proBNP levels in HFpEF and HFrEF}

That patients with HFpEF more often have lower NT-proBNP and BNP levels than patients with HFrEF, at similar levels of end-diastolic left ventricular pressure, is explained by the correlation of natriuretic peptide levels with diastolic wall stress, which is lower for smaller left ventricular volumes and thicker left ventricular walls, which are both hallmarks for patients with HFpEF. ${ }^{27}$ The similar improvements in percentage reductions in NT-proBNP in HFpEF and HFrEF may be somewhat unexpected, since there are less prognostically beneficial therapies for HFpEF than for HFpEF. At discharge, there were no significant differences in the prescription of beta-blockers or diuretics between HFpEF and HFrEF patients, while ACE inhibitors/ARBs were less often prescribed in HFpEF. We know that ARBs do not affect NT-proBNP levels in patients with HFpEF, ${ }^{30}$ and another explanation must be found why NT-proBNP reductions were as large in patients with $\mathrm{HFpEF}$ as in patients with HFrEF. The significantly higher SBP is notable among patient with HFpEF, which may have given the clinicians more opportunity to increase the dosage of diuretics during hospitalisation in HFpEF patients.

A strategy that uses absolute NT-proBNP values as risk thresholds and uses relative reductions in NT-proBNP as targets may benefit from the finding that both patient groups will be able to reach realistic but still prognostically important reductions in NT-proBNP. As for outcome, cardiovascular outcomes may be predicted by natriuretic peptides in HFpEF and HFrEF, but this study and other studies demonstrate that for patients with HFpEF with low NT-proBNP levels outcome improvements

Table 4 Distribution of risk factors according to NT-proBNP levels at discharge and heart failure groups

\begin{tabular}{|c|c|c|c|c|c|c|}
\hline & \multicolumn{3}{|c|}{$\begin{array}{l}\text { NT-proBNP at discharge } \leq 3000 \mathrm{pg} / \mathrm{mL} \\
(\mathrm{n}=580)\end{array}$} & \multicolumn{3}{|c|}{$\begin{array}{l}\text { NT-proBNP at discharge }>3000 \mathrm{pg} / \mathrm{mL} \\
(\mathrm{n}=591)\end{array}$} \\
\hline & HFpEF & HFrEF & P value & HFpEF & HFrEF & $P$ value \\
\hline Age $\geq 75$ years, $n(\%)$ & $101(58)$ & $92(29)$ & $<0.001$ & $64(67)$ & $199(48)$ & 0.001 \\
\hline Ischaemic aetiology, $n(\%)$ & $97(63)$ & $147(51)$ & 0.027 & $43(54)$ & $174(44)$ & 0.108 \\
\hline Peripheral oedema at admission, $\mathrm{n}(\%)$ & $100(63)$ & $162(54)$ & 0.060 & $66(73)$ & $255(65)$ & 0.218 \\
\hline $\mathrm{SBP} \leq 115 \mathrm{~mm} \mathrm{Hg}$ at admission, $\mathrm{n}(\%)$ & $27(16)$ & $84(26)$ & 0.007 & $24(26)$ & $188(45)$ & $<0.001$ \\
\hline Atrial fibrillation at admission, $\mathrm{n}(\%)$ & $85(51)$ & $110(37)$ & 0.005 & $50(57)$ & $162(42)$ & 0.017 \\
\hline Anaemia at admission, $\mathrm{n}(\%)$ & $77(46)$ & $129(41)$ & 0.290 & $59(63)$ & $241(60)$ & 0.640 \\
\hline Hyponatraemia $(<135 \mathrm{mmol} / \mathrm{L})$ at admission, $\mathrm{n}(\%)$ & $19(14)$ & $34(11)$ & 0.435 & $18(21)$ & $94(23)$ & 0.778 \\
\hline Serum urea $\geq 15 \mathrm{mmol} / \mathrm{L}$ at admission, $\mathrm{n}(\%)$ & $14(11)$ & $31(11)$ & 1.000 & $32(41)$ & $145(39)$ & 0.899 \\
\hline Comorbidities $\leq 2, \mathrm{n}(\%)$ & $69(63)$ & $196(76)$ & 0.011 & $28(39)$ & $134(40)$ & 0.895 \\
\hline Comorbidities >2, n (\%) & 41 (37) & $62(24)$ & & $44(61)$ & $199(60)$ & \\
\hline
\end{tabular}

HFpEF, heart failure with preserved ejection fraction; HFrEF, heart failure with reduced ejection fraction; NT-proBNP, N-terminal pro-B-type natriuretic peptide; SBP, systolic blood pressure. 
should probably also be sought in therapies targeting non-cardiovascular outcomes. Alternatively, when natriuretic peptides levels are to be used as surrogate endpoints, these endpoints should be better defined as cardiovascular endpoints.

\section{LIMITATIONS}

Variation in NT-proBNP assays used should be considered. Nevertheless, this range in markers reflects the day-to-day clinical practice. The relatively small sample size of patients with $\mathrm{HFpEF}$ in the cohort compared with that of HFrEF patients should be considered, with possible impact on the statistical significance of the used NT-proBNP categories. We did correct for the bias from data missing at random by using multiple imputation pooling algorithms. The endpoint of all-cause mortality is limiting, as cardiac markers should be best used for cardiac outcomes. It is clear that CV mortality explains $63 \%-87 \%$ of mortality after ADHF. ${ }^{3132}$ In this respect, a previous study has shown that the proportion of mortality in heart failure explained by cardiac and non-cardiac causes is the same in HFpEF as in HFrEF but maybe also because the comorbidities were more evenly distributed between HFpEF and HFrEF than in other studies. ${ }^{31}$

\section{CONCLUSIONS}

Our study suggests that there is no difference between patients with HFpEF and HFrEF in relative risk prediction of 6-month mortality by absolute discharge NT-proBNP levels or by percentage NT-proBNP changes. To explain the similar

\section{Key messages}

\section{What is already known on this subject?}

- Prognostic information of a single measurement of natriuretic peptide levels has been specifically investigated and reported to be equal for both heart failure with preserved ejection fraction (HFpEF) and heart failure with reduced ejection fraction (HFrEF) either at admission or at discharge.

\section{What might this study add?}

- This study adds to the present knowledge that the percentage changes in N-terminal pro-B-type natriuretic peptide (NTproBNP) levels during hospitalisation and absolute discharge NT-proBNP values are contributing prognostic information for 6 months without the need to distinguish between patients with HFpEF and HFrEF. Our study further highlights the possibility that comorbidities contribute relatively more to prognosis in patients with HFpEF with lower NT-proBNP levels than in patients with HFrEF with lower NT-proBNP levels. Finally, despite known treatment obstacles in patients with HFpEF, our study shows that patients with HFpEF reach similar or larger relative reductions in NT-proBNP compared with patients with HFrEF.

\section{How might this impact on clinical practice?}

- A strategy that uses absolute NT-proBNP values as risk thresholds and uses relative reductions in NT-proBNP as targets may benefit from the finding that both patient groups will be able to reach realistic but still prognostically important reductions in NT-proBNP. This study and other studies demonstrate that for patients with HFpEF with low NT-proBNP levels, outcome improvements should probably also be sought in therapies targeting non-cardiovascular outcomes. long-term mortality in HFpEF as in HFrEF, despite lower NT-proBNP discharge levels in HFpEF, we raise the possibility that the larger burden of prognostic relevant comorbidities in patients with HFpEF with low NT-proBNP levels unfavourably affects their prognosis.

Correction notice This article has been corrected since it first published online. The open access licence type has been amended.

Contributors KS, WEK and YMP had the idea for the project, designed the collaborative analysis and undertook searches of published work. KS and SS collected the individual patient data and prepared for analysis. Statistical analysis and elaboration of figures was done by KS, WEK and JGPT. KS wrote the paper with important contribution from WEK, SS and YMP and input from MM. LWE, AB-G, JGPT, MM and VV provided valuable comments on the report. All principal investigators shared individual patient data and had an opportunity to contribute to the interpretation of results and to the redrafting of the report. All authors reviewed and revised the manuscript and approved the final version.

Funding LWE and YMP received research funding for their original study from the Dutch Heart Foundation, Dutch Organisation for Scientific Research (NWO), the Roya Dutch Academy of Arts and Sciences (KNAW) - Interuniversity Cardiology Institute of the Netherlands, Pfizer, Astra-Zeneca, Medtronic and Roche Diagnostics.

Competing interests YMP is a recipient of payments for lectures including service on speakers' bureaus and research grants from Roche Diagnostics. YMP has an unrelated biomarker patent and stocks in a university spinoff company. WEK received a grant (2010B97) from the Dutch Heart Foundation for the PRIMA II study and has participated in advisory board meetings of Roche Diagnostics and Novartis. MM is a member of the board in Corthera and Novartis and receives payment for lectures including service on speakers' bureaus from Servier or Stroder.

\section{Patient consent for publication Not required.}

Ethics approval All studies were approved by the ethical committees in their respective centres. ${ }^{11182021}$

Provenance and peer review Not commissioned; externally peer reviewed

Open access This is an open access article distributed in accordance with the Creative Commons Attribution 4.0 Unported (CC BY 4.0) license, which permits others to copy, redistribute, remix, transform and build upon this work for any purpose, provided the original work is properly cited, a link to the licence is given, and indication of whether changes were made. See: https://creativecommons.org/ licenses/by/4.0/.

\section{REFERENCES}

1 Fonarow GC, Stough WG, Abraham WT, et al. Characteristics, treatments, and outcomes of patients with preserved systolic function hospitalized for heart failure: a report from the OPTIMIZE-HF Registry. J Am Coll Cardiol 2007;50:768-77.

2 Yancy CW, Lopatin M, Stevenson LW, et al. Clinical presentation, management, and in-hospital outcomes of patients admitted with acute decompensated heart failure with preserved systolic function. J Am Coll Cardiol 2006;47:76-84.

3 Senni M, Gavazzi A, Oliva F, et al. In-hospital and 1-year outcomes of acute heart failure patients according to presentation (de novo vs. worsening) and ejection fraction. Results from IN-HF Outcome Registry. Int J Cardiol 2014;173:163-9.

4 Bhatia RS, Tu JV, Lee DS, et al. Outcome of heart failure with preserved ejection fraction in a population-based study. N Engl J Med 2006;355:260-9.

5 Owan TE, Hodge DO, Herges RM, et al. Trends in prevalence and outcome of heart failure with preserved ejection fraction. N Engl J Med 2006;355:251-9.

6 Kang SH, Park JJ, Choi DJ, et al. Prognostic value of NT-proBNP in heart failure with preserved versus reduced EF. Heart 2015;101:1881-8.

7 van Veldhuisen DJ, Linssen GC, Jaarsma T, et al. B-type natriuretic peptide and prognosis in heart failure patients with preserved and reduced ejection fraction. J Am Coll Cardiol 2013;61:1498-506.

8 Lam CSP, Gamble GD, Ling LH, et al. Mortality associated with heart failure with preserved vs. reduced ejection fraction in a prospective international multi-ethnic cohort study. Eur Heart J 2018;39:1770-80.

9 Meta-analysis Global Group in Chronic Heart Failure (MAGGIC). The survival of patients with heart failure with preserved or reduced left ventricular ejection fraction: an individual patient data meta-analysis. Eur Heart J 2012;33:1750-7.

10 Kociol RD, Horton JR, Fonarow GC, et al. Admission, discharge, or change in B-type natriuretic peptide and long-term outcomes: data from Organized Program to Initiate Lifesaving Treatment in Hospitalized Patients with Heart Failure (OPTIMIZE-HF) linked to Medicare claims. Circ Heart Fail 2011;4:628-36.

11 Salah K, Kok WE, Eurlings LW, et al. A novel discharge risk model for patients hospitalised for acute decompensated heart failure incorporating N-terminal pro-Btype natriuretic peptide levels: a European coLlaboration on Acute decompeNsated Heart Failure: ELAN-HF Score. Heart 2014;100:115-25. 
12 Bettencourt P, Azevedo A, Pimenta J, et al. N-terminal-pro-brain natriuretic peptide predicts outcome after hospital discharge in heart failure patients. Circulation 2004; 110:2168-74.

13 Bettencourt P, Azevedo A, Fonseca L, et al. Prognosis of decompensated heart failure patients with preserved systolic function is predicted by NT-proBNP variations during hospitalization. Int J Cardiol 2007;117:75-9.

14 Paul B, Soon KH, Dunne J, et al. Diagnostic and prognostic significance of plasma $\mathrm{N}$-terminal-pro-brain natriuretic peptide in decompensated heart failure with preserved ejection fraction. Heart Lung Circ 2008;17:497-501.

15 Valle R, Aspromonte N, Feola M, et al. B-type natriuretic peptide can predict the medium-term risk in patients with acute heart failure and preserved systolic function. J Card Fail 2005;11:498-503.

16 Anand IS, Rector TS, Cleland JG, et al. Prognostic value of baseline plasma aminoterminal pro-brain natriuretic peptide and its interactions with irbesartan treatment effects in patients with heart failure and preserved ejection fraction: findings from the I-PRESERVE trial. Circ Heart Fail 2011;4:569-77.

17 Metra M, Nodari S, Parrinello G, et al. The role of plasma biomarkers in acute heart failure. Serial changes and independent prognostic value of NT-proBNP and cardiac troponin-T. Eur J Heart Fail 2007:9:776-86.

18 Salah K. NT-proBNP as a risk stratification tool for the management of acute decompensated heart failure, 2018.

19 O'Donoghue M, Chen A, Baggish AL, et al. The effects of ejection fraction on $\mathrm{N}$-terminal ProBNP and BNP levels in patients with acute CHF: analysis from the ProBNP Investigation of Dyspnea in the Emergency Department (PRIDE) study. J Card Fail 2005;11:S9-14.

20 Stienen S, Salah K, Moons AH, et al. Rationale and design of PRIMA II: A multicenter, randomized clinical trial to study the impact of in-hospital guidance for acute decompensated heart failure treatment by a predefined NT-PRoBNP target on the reduction of readmIssion and Mortality rAtes. Am Heart J 2014;168:30-6.

21 Stienen S, Salah K, Moons AH, et al. NT-proBNP (N-Terminal pro-B-Type Natriuretic Peptide)-Guided Therapy in Acute Decompensated Heart Failure: PRIMA II Randomized Controlled Trial (Can NT-ProBNP-Guided Therapy During Hospital Admission for Acute Decompensated Heart Failure Reduce Mortality and Readmissions?). Circulation 2018;137.

22 Ponikowski P, Voors AA, Anker SD, et al. 2016 ESC Guidelines for the diagnosis and treatment of acute and chronic heart failure: The Task Force for the diagnosis and treatment of acute and chronic heart failure of the European Society of Cardiology (ESC). Developed with the special contribution of the Heart Failure Association (HFA) of the ESC. Eur J Heart Fail 2016;18:891-975.

23 Stienen S, Salah K, Eurlings LW, et al. Challenging the two concepts in determining the appropriate pre-discharge $\mathrm{N}$-terminal pro-brain natriuretic peptide treatment target in acute decompensated heart failure patients: absolute or relative discharge levels? Eur J Heart Fail 2015;17:936-44.

24 Grambsch PM, Therneau TM. Proportional hazards tests and diagnostics based on weighted residuals. Biometrika 1994;81:515-26.

25 Michtalik HJ, Yeh HC, Campbell CY, et al. Acute changes in N-terminal pro-B-type natriuretic peptide during hospitalization and risk of readmission and mortality in patients with heart failure. Am J Cardiol 2011;107:1191-5.

26 Maeder MT, Rickenbacher P, Rickli H, et al. N-terminal pro brain natriuretic peptide-guided management in patients with heart failure and preserved ejection fraction: findings from the Trial of Intensified versus standard medical therapy in elderly patients with congestive heart failure (TIME-CHF). Eur J Heart Fail 2013;15:1148-56

27 Iwanaga Y, Nishi I, Furuichi S, et al. B-Type natriuretic peptide strongly reflects diastolic wall stress in patients with chronic heart failure. J Am Coll Cardiol 2006;47:742-8.

28 Komajda M, Carson PE, Hetzel S, et al. Factors associated with outcome in heart failure with preserved ejection fraction: findings from the Irbesartan in Heart Failure with Preserved Ejection Fraction Study (I-PRESERVE). Circ Heart Fail 2011:4:27-35.

29 Mentz RJ, Kelly JP, von Lueder TG, et al. Noncardiac comorbidities in heart failure with reduced versus preserved ejection fraction. J Am Coll Cardiol 2014:64:2281-93.

30 McKelvie RS, Komajda M, McMurray J, et al. Baseline plasma NT-proBNP and clinical characteristics: results from the irbesartan in heart failure with preserved ejection fraction trial. J Card Fail 2010;16:128-34.

31 Hamaguchi S, Kinugawa S, Sobirin MA, et al. Mode of death in patients with heart failure and reduced vs. preserved ejection fraction: report from the registry of hospitalized heart failure patients. Circ J 2012;76:1662-9.

32 O'Connor CM, Miller AB, Blair JE, et al. Causes of death and rehospitalization in patients hospitalized with worsening heart failure and reduced left ventricular ejection fraction: results from Efficacy of Vasopressin Antagonism in Heart Failure Outcome Study with Tolvaptan (EVEREST) program. Am Heart J 2010;159:841-9. 